\title{
Coding for Distributed Computation
}

\author{
Leonard J. Schulman* \\ Computer Science Division \\ U. C. Berkeley \\ Berkeley CA 94720
}

Extended communications among component processors are essential to the operation of all but the simplest computers. In this talk we are concerned with the following question: if the communications among processors, linked in some network, are unreliable, what is the effect on the efficiency and reliability with which the network can perform a computation?

An important case of this scenario, that in which there are two processors and the required task is to transmit a large block of data from one to the other, actually predates large-scale computing. Shannon's coding theorem addresses this problem, and shows that in order to reliably transmit a message of $T$ bits over a noisy communication channel it suffices to send a message of length $T \frac{1}{C}$ (for $0<C<1$ the "Shannon capacity" of the channel). The theorem ensures that the probability of a decoding error is exponentially small in the message length $T$.

We will describe analogous coding theorems for the more general, interactive, communications required in computation. In this case the bits transmitted in the protocol are not known to the processors in advance but are determined dynamically. Therefore the block encoding technique used in the proof of Shannon's theorem, does not apply.

First we show that any interactive protocol of length $T$ between two processors connected by a noiseless channel can be simulated, if the channel is noisy (a binary symmetric channel of capacity

\footnotetext{
*Research supported by an NSF Mathematical Sciences Postdoctoral Fellowship.
}

$C$ ), in time proportional to $T \frac{1}{C}$, and with error probability exponentially small in $T$.

Then we show that this result can be extended to arbitrary distributed network protocols. We show that any distributed protocol which runs in time $T$ on a network of degree $d$ having noiseless communication channels, can, if the channels are in fact noisy, be simulated on that network in time proportional to $T \frac{1}{C} \log d$. The probability of failure of the protocol is exponentially small in $T$.

Preliminary presentations of these results can be found in $[1,2]$.

The network theorem is joint with Sridhar Rajagopalan.

\section{References}

[1] S. Rajagopalan and L. J. Schulman. Coding for distributed computation. In Proceedings of the 26th Annual Symposium on Theory of Computing, 1994.

[2] L. J. Schulman. Deterministic coding for interactive communication. In Proceedings of the 25th Annual Symposium on Theory of Computing, pages 747-756, 1993. 